\title{
Weighted Impact and Ranking Model (Wirm) For Airport Con- gestion
}

\author{
Rupali Dahake ${ }^{1}$, Dr. G Prasanna Lakshmi ${ }^{2}$ \\ ${ }^{1}$ Research Scholar, Pacific University, Udaipur \\ ${ }^{2}$ Guide, (WOS-A) Andhra University.
}

\begin{abstract}
This study aims to figure out one of the current challenges to aviation is reducing the congestion in major airports. The problem statement describes an important issue for system planners, airport operators and management who are dealing with various aspect of congestion at airport. At the same time resource optimization is a primary concern for all segments in the system. The proposed model offers a specialized Multi Criteria Decision making model for smooth and manageable passenger flow process at airport. It also focus on passenger operation and minimum service time by forecasting various factors that affect to down the performance, efficient use of resources. The proposed model is able to serve dynamic solution by forecasting the undesirable condition as per different timeframe.
\end{abstract}

Keywords: Decision variables, Multi-Criteria Decision Making, ranking, timeframe

\section{Introduction}

In the information era, there is growing notion of "Big-data" which is increasing exponentially in the terms of Volume, Velocity, Variety, Veracity and Value. We believe that appropriate investment in big data will results into technological aspects of advance big data management in next generation.

Thomas Davenport stated that "There is considerable evidence that decision based on analytics are more like to be correct than those based on intuition".

Matthias Ehrgott et al discussed that, decision-making analysis provides revolutionary and promising approach to big data Analytics as demand of digital world. It is more challenging and visionary to deriving gigantic data. The subject of multi-criteria optimization is the selecting good decision from a set of alternatives with respect to multi criteria or objective functions.

In all types of transportation, especially air transport commits to minimize time and have more comfortable journey. In this scenario, major modifications are required on existing working procedure at airport. Proposed idea considers few most important factors that are directly correlated with all probabilities of congestions at airport. We named as decision variables. By using multi criteria decision making analysis; we are optimizing solution, which give promising results.

\section{Optimization techniques}

\section{Overview Of Studies}

Optimization is the act of obtaining the best result under the given circumstances to minimize the effort required and to maximize the desired benefit .The optimization process is a systematic way by using various constraints and criteria to identify the optimal solution, which help into factor analysis.

DNagesh Kumar presented that hill climbing is an advance optimization technique which uses an iterative algorithm that starts with an arbitrary solution to a problem then attempts to find a better solution by incrementally changing a single element of the solution. Likewisestochastic hill climbing does not examine all neighbors before deciding how to move. Ratherit selects a neighbor at random, and decides (based on the amount of improvement in that neighbor) whether to move to that neighbor or to examine another. Hamsa Balakrishnan (2016) was discussed problems of air transportation systems and provides Airport congestion control, Large-scale optimization algorithms for air traffic flow management and enhanced system with its robustness and safety which is implemented by ATFM algorithm.

Problems of runway scheduling and taxiway routing simultaneously for both departure and arrival aircrafts is resolved by Chuhang Yu et al (2015).Solution is offered by a set partitioning model with side constraint in which each possible aircraft route in the taxiway and runway is referred as a decision variable.

In the recent studies, Jaromir et al (2013) was created functional model and analyzed in the extent of available data necessary for the model calibration. Model is usable also for another analysis in the future, because the input data could beeasily changed. It is also possible to change model structure, so that it could be used also forevaluating of passenger flow processes in another airport terminal. 


\section{Multi-criteria Decision Making}

In order to derive precious information, significant improvements have been made over the last decade in the sense of Multi-criteria Decision Making. There are different classification of MCDM problems and methods based on whether the solutions are explicitly or implicitly defined. When we are discussing this approach to resolve the problem of Airport congestion, one can definitely forecast the future of airport in coming year that is multiple accesses of airport resources which consequently raise the problem of congestion at airport. With the supporting methodology ELECTREToni (2015) had implemented new fuzzy MCDM approach, which gives strategic benchmarking of service quality for international airport in Sicily (Italy).Nizamuddin et al (2015) presented use of (AHP) Analytic Process Model, whichallow multi-objective problem analysis to facilitate decision making only who are seeking for multi criteria approached. Milan et al (2002) integrated three discrete MCDM methods as SAW (Simple Additive Weighting), TOPSIS (Technique for Order Preference by Similarity to the Ideal Solution) and AHP (Analytic Hierarchy Process) to define framework which focus specific number of alternatives and attributes for decision making.

When application demand decision making based on more than two criteria, Paul, Franz (2009) invented new additivemulti attributes method with the basic concept of Potentially All Pairwise Ranking of all possible Alternatives (PAPRIKA).This method allow to combine multiple alternatives to get optimized solution for decision maker.

Later in Anjali Awasthi et al (2011) developed new fuzzy theory model in a multi-criteria decision making approach in location planning for urban distribution center Due to lack of data uncertainty directly affecting selection of best location.. And purpose of this model was to achieve sensitive analysis to prevent the influence of criteria weights in the form of decision making.

Miriam et al (2012) formulated the model, based on fuzzy TOPSIS, ANP and fuzzy DEMATEL to highlight air traffic at Philippines. The result indicates that suitable set of action are applied to improve the degree of safety. And decision making perform on basis of safety criteria.Furthermore, Nattapong (2015) implemented and improved classic solution to establish airport hubs using multiple criteria decision making. Evolutionary approach TOPSIS and analytic Hierarchy Process (AHP) work together to analyzed influencing factor for airport establishment. On the other hand, proposed implication offered solution by Delphi method and Regression analysis function

Slavica et al(2015) presented integration of Analytic Hierarchy Process (AHP) and the Even Swaps Method (ESM) MCDM methods to study regional airline. The results revealed that both sensitive analysis and objective ranking almost equally good as the aircraft type selection in multi criteria decision making.

In recent studies, Assem El-Ansary (2015) developed interactive software toolnamed as DECISION for critical event analysis. Tool identifies effectiveness criteria of the Analytic Hierarchy Process (AHP) and the ELECTRE Pair wise Comparison approaches. In analysis model any two factors are compared among the set of object, criteria and events.

\section{Introduction}

\section{Weighted Impact And Ranking Model (Wirm)}

The Weighted Impact and Ranking Model (WIRM) have two datasets which is known as Airlines and Decision variables. The decision variables are nothing but factors which results into the problem of airport congestions. Like Peak-hours (Time at which more passenger at airport), Natural calamities (E.g. Rain, Fog), Group Tour passenger, Interval between two connected flights etc. Now we are considering impact of these decision variables in the form of Low, Medium and High and allocate weightage to impact. Now total time is divided into two T1 (12 hours.) and T2 (12 hours.) as a day and Night respectively. Observe the Table 1 for some declaration of WIRM Model.

\section{Formulae:}

Consider, Total Weightage of Impact $=100$

Total W $(\mathrm{I})=$ Low + Medium + High

$100=(x+1)+2 x+6 x$

\begin{tabular}{|l|l|l|l|l|}
\hline Air- lines & Decision Variables & Impact & Weightage & Time frame \\
\hline A & D & I & W(I) & T \\
\hline A1 & D1 & Low & 12 & T1(Day) \\
\hline A2 & D2 & Medium & 22 & T2(Night) \\
\hline. &. & High & 66 & \\
\hline. &. & & & \\
\hline. &. & & & \\
\hline. &. & & & \\
\hline An & Dm & & & \\
\hline
\end{tabular}

Table1: Declaration of parameters of WIRM Model and their possible values. 
Component Diagram

The workflow of Weighted Impact and Ranking Model (WIRM) can be shown in detail as following component diagram.

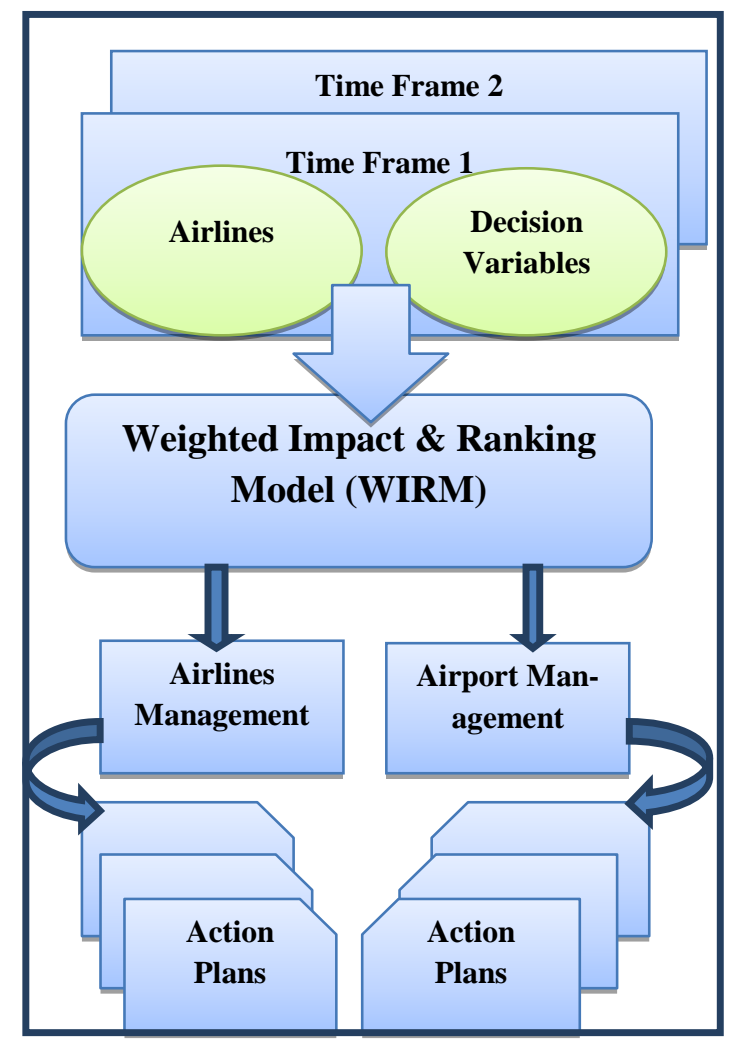

Fig. 1 Component Diagram of WIRM

\section{Working}

Step 1: Give Input to model

$$
\mathrm{A}=\{1,2,3 \ldots \ldots \mathrm{n}) \text {. }
$$

Where $\mathrm{n}=$ Total Number of Airline at the Airport.

$$
\mathrm{D}=\{1,2,3 \ldots \mathrm{m}) \text {. }
$$

Where $m=$ total Number Decision variables considered.

Step 2: Design Matrix for each A and T1 by considering D and W (I).

We need to considertable2as sample matrix representation of Decision variables and Weightage.

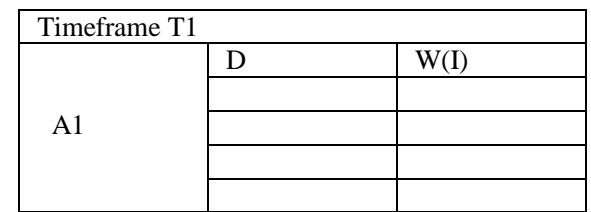

Table2: Matrix Representation

Step 3: Now, $X=$ Combine matrix for all A.

Consider Table3 as finalsample matrix for all airlines A in timeframe T1.

\begin{tabular}{|l|l|l|l|l|}
\hline \multirow{4}{*}{} & \multicolumn{4}{|l|}{ Weightage W (I) } \\
\hline & A1 & A2 & & An \\
\hline D1 & & & & \\
\hline D2 & & & & \\
\hline. & & & & \\
\hline. & & & & \\
\hline. & & & & \\
\hline Dm & & & & \\
\hline
\end{tabular}

Table3: Matrix Representation 
Step 4: Performs sum of rows to Matrix $-\mathrm{X}$

$\mathrm{X}=\left(x_{i j}\right)$

Where $i=(1,2,3 \ldots \mathrm{m})$ and

$j=(1,2,3 \ldots . \mathrm{n})$

Sum of rows $(i)=\sum_{j=1}^{n}\left(x_{i j}\right)$

Step 5: Perform ranking by applying sorting on output of step 4.

Step 6: Highlight topresults. And provide solution set for Airport management

Step 7: Perform sum of columns of Matrix X

Sum of Columns $(\mathrm{j})=\sum_{i=1}^{m}\left(x_{i j}\right)$

Step 8: Perform ranking by apply sorting on step 7

Step 9: Highlight topresults. And provide solution set for Airline management

Step 10: Repeat step 2 to 9 for time frame T2.

\section{Conclusion}

In the paper, proposed result revealed that model is able find out real impact of various root causes in problem of airport congestion. In particular, we see that it looks like a generalized model because input could be easily changed and have potential to analyses the multiple criteria for appropriate decision making to avoid traffic at airport in advance.

An outstanding part of this model is, it magnifies highly affecting factors of airport traffic and judgmental decision to which we can suggest solutions either to particular airline management or airport managements. Further studies will include selection of cost effective selection of solution set. And feasibility condition of system implementation, big data analytic methodology.

\section{References}

[1]. Matthias Ehrgott and XavierGandibleux "Multiple Criteria Optimization: State of the Art Annotated Bibliographic Surveys"

[2]. Jaromir Siroky, Pavlina Hlavsova 2014. Optimizing process of check-in and security check at airport terminals. Number 4, Volume IX, December.

[3]. D Nagesh Kumar, IISc Optimization Methods: M1L1.

[4]. Ishikawa, Kaoru (1976). Guide to Quality Control. Asian Productivity Organization. ISBN 92-833-1036-5.

[5]. Da Silva de Santis SH, Marcicano JPP, Cunto G, Figueiredo RA (2016) .Use of Quality Tools for Problem Analysis (FMEA and Ishikawa Diagram) in a Small Textile Business Textile Sci Eng 6: 258. doi:10.4172/2165-8064.1000258.

[6]. En.wikipedia.org Wikipedia contributors. Hill climbing. Wikipedia, the Free Encyclopedia. Wikipedia.

[7]. Chuhang Yu and Henry Y. K. Lau, the University of Hong Kong, Hong Kong. Integrated Optimization of Airport Taxiway and Runway Scheduling.

[8]. Hamsa Balakrishnan. Control and Optimization Algorithms for Air Transportation Systems. Department of Aeronautics and Astronautics Massachusetts Institute of Technology Cambridge, MA 02139, USA.

[9]. Vasile Dumbravă, Titu Maiorescu. Using Probability - Impact Matrix in Analysis and Risk Assessment Projects. University of Bucharest, Romania; Vlăduț - Severian Iacob, Stefan cel Mare University of Suceava, Romania -2013.

[10]. Guizzi g., Murino t., Romano e. A Discrete Event Simulation to model Passenger Flow in the Airport Terminal. Department of Material Engineering and Production University of Naples "Federico II" Piazzale Tecchio, 80 - 80125 Napoli ITALY.

[11]. Raghavendra Kune, Pramod Kumar Konugurthi, Arun Agarwal2. The Anatomy of Big Data Computing.

[12]. http://www.mcdmsociety.org, 18.12.2008.

[13]. multi criteria decision making: a case of locating low cost carrier terminal (air) in malaysianizamuddin zainuddin, dayang shalbia Abdulghani, Adam mohd saifudin. Journal of Technology and Operations Management 10(1), 23-38 (2015).

[14]. Toni Lupo. Benchmarking of airport service quality by A new fuzzy MCDM approach “, European transport Issue 57,paper ISSN 1825-3997

[15]. Milan Janic and Aura Reggiani. An Application of the Multiple Criteria Decision Making (MCDM) Analysis to the Selection of a New Hub Airport EJTIR, 2, no. 2 (2002), pp. 113

[16]. Paul Hansen, Franz Ombler. A New Method of Scoring additive Multi-attribute value Models Using Pairwise Ranking of Alternatives. Journal of Multi-criteria Decision analysis 2009

[17]. Anjali Awasthi a, S.S. Chauhanb, S.K. Goyal 2011. A multi-criteria decision making approach for location planning for urban distribution centers under uncertainty. Mathematical and Computer Modelling 53 (2011) 98-109

[18]. Miriam F. BongoLanndon Ocampo.2012.A Hybrid Fuzzy Multi-Criteria Decision-Making Approach for Mitigating Air Traffic Congestion.

[19]. Nattapong Jantachalobon. The evaluation forms of international passenger airport hub in Southeast Asia. School of Engineering, Bangkok, Thailand N doi:10.5937/jaes13-8382-2015

[20]. Slavica Dozic Milica Kalic.2015.Comparison of Two MCDM Methodologies in Aircraft Type Selection Problem. Transportation Research ProcediaVolume 10, 2015, Pages 910-919

[21]. Assem El-Ansary 2015.Requirements Definitions of Real-Time System Using the Behavioral Patterns Analysis (BPA) Approach: The Air Traffic Control System" Vol. 3, No. 2, May 2015.

[22]. www.wikipedia.org 


\section{Biography:}

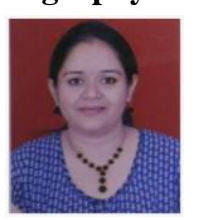

Mrs. Rupali Dahake passed M.Sc. (Computer Science) from North Maharashtra University, Jalgaon. Currently she is pursuing her Ph.D. from Pacific University, Udaipur.

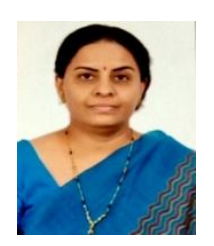

Dr. G.Prasanna Lakshmi. Guide. Women Scientist (WOS-A) Andhra University. 